\title{
Future Trends of Mesothelioma Mortality in Japan Based on a Risk Function
}

\author{
Tomoya MYOJIN ${ }^{1}$, Kenichi AZUMA ${ }^{2 *}$, Jiro OKUMURA ${ }^{2}$ and Iwao UCHIYAMA ${ }^{3}$ \\ ${ }^{1}$ Department of Community Health and Preventive Medicine, Hamamatsu University School of Medicine, Japan \\ ${ }^{2}$ Department of Environmental Medicine and Behavioral Science, Kinki University Faculty of Medicine, Japan \\ ${ }^{3}$ Emeritus Professor, Kyoto University, Japan
}

Received January 31, 2010 and accepted January 31, 2012

Published online in J-STAGE March 28, 2012

\begin{abstract}
Mesothelioma is a malignancy with poor prognosis. It is chiefly caused by asbestos exposure and its symptoms can occur about 30-50 yr after the initial exposure. This study aims to predict the future trends in mesothelioma mortality in Japan using a method that is an alternative to the age-cohort model. Our approach is based on a risk function that links mesothelioma mortality combined with data pertaining to the population, size of the labor force, and quantity of asbestos imports. We projected the number of deaths occurring in individuals aged 50-89 for yr 2003-2050 using risk functions. Our results have indicated that mesothelioma mortality among Japanese people aged 50-89 yr will continue to increase until 2027 and reach a maximum of 66,327 deaths in the years 2003-2050. Our estimate has also suggested that the number of mesothelioma deaths could be significantly reduced if there were adequate compliance with the administrative level guidelines for occupational asbestos exposure.
\end{abstract}

Keywords: Asbestos, Mesothelioma, Mortality, Risk function, Exposure

\section{Introduction}

Mesothelioma is a malignancy that develops in mesothelial cells and has a poor prognosis. The survival time of mesothelioma ranges between several months to years. This malignancy is induced by asbestos or erionite. Those causalities have been epidemiologically confirmed $^{1)}$. As erionite has been used sparingly as a specimen of mineral in Japan, mesothelioma cases are chiefly attributed to asbestos exposure. The symptoms can occur about 30-50 yr after the first exposure. The statistics of mesothelioma mortality in Japan have been recorded since $1995^{2)}$. In 2009, 1156 patients died of mesothelioma. The number

*To whom correspondence should be addressed.

E-mail: kenazuma@med.kindai.ac.jp

(C)2012 National Institute of Occupational Safety and Health of victims is increasing every year since 2000 when more than 710 deaths were recorded. Mesothelioma has various forms (e.g., excessive platelets, coelomic fluid accumulation, pectoralgia) thus complicating the diagnosis even for an expert. The diagnostic technology has advanced and the immunohistological staining technique has been implemented in Japan since the early 1990s.

The trends in asbestos imports and the mortality of mesothelioma are shown in Fig. 1. Asbestos imports resumed after World War II, and increased sharply in correlation with the economic development in Japan. For instance, in the 1970 s and 1980 s, more than 200,000 tons of asbestos was imported annually ${ }^{3)}$. Hence, we believe that the number of mesothelioma deaths are likely to further increase in future.

The Ministry of the Environment (MOE) ${ }^{4)}$ and $\mathrm{Mu}-$ rayama et al. ${ }^{5)}$ had independently predicted the future 


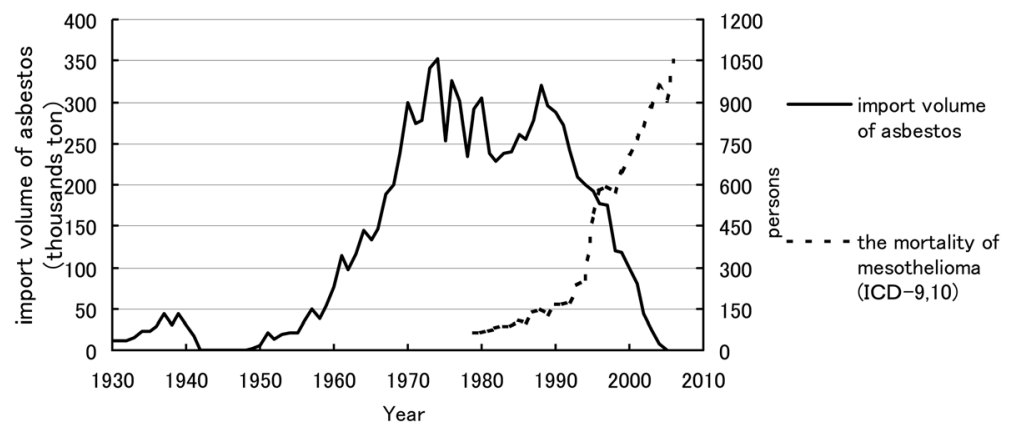

Fig. 1. Asbestos imports and the mortality of mesothelioma.

trends in mesothelioma mortality. The MOE analyzed the relation between mesothelioma deaths and asbestos imports in 11 countries based on the Tossavainen ${ }^{6)}$ report. The MOE reported 1 mesothelioma death per 170 tons of asbestos imported. A total of 9.6 million tons of asbestos was imported by Japan from 1949 to 2006. The MOE has estimated mesothelioma mortality to approach 60,000 in future ${ }^{4)}$.

Murayama et al. estimated projections based on an agecohort model, which estimated the trends in mesothelioma mortality from population projections for Japan by estimating some parameters using a log-linear model based on the death rate and a Poisson distribution. These estimates predicted an average of 58,800 and 103,000 mesothelioma deaths by 2029 and 2039, respectively ${ }^{5}$.

Most mesothelioma cases have been attributed to occupational asbestos exposure in most patients. However, the predictions for mesothelioma trends cited previously did not sufficiently reflect actual working conditions and failed to consider the actual data (e.g., the amount of asbestos imported and working population size). The extent of asbestos exposure has varied from year to year correlating with the quantity of asbestos that was imported (Fig. 1). Based on those insights, the aim of this study is to project the future trends in mesothelioma mortality based on a risk function that takes into the following parameters specific to Japanese workers: the number of mesothelioma deaths in past years, the size of the labor force, age at exposure and the quantity of imported asbestos.

\section{Materials and Methods}

\section{Risk estimation model}

Banaei et al. predicted French future trends in mesothelioma mortality with risk functions ${ }^{7}$. The Banaei model in equation 1 is based on past epidemiological studies ${ }^{8-11)}$.
$R(x)=K_{e} \sum_{a=0}^{a=x} c(a)(x-a)^{P_{e}}+K_{b} x^{P_{b}}$

$x$ : age at death of mesothelioma

$R(x)$ : risk of dying from mesothelioma at age $x$

$K_{e}$ : the coefficient of mesothelioma death related to asbestos exposure

$P_{e}$ : the exponent of age at mesothelioma death related to asbestos exposure

$a$ : the variant of age

$c(a)$ : exposure concentration at age $a$

$K_{b}$ : the coefficient related to mesothelioma death of spontaneous onset

$P_{b}$ : the exponent related to age at mesothelioma death of spontaneous onset

The left side of the equation $R(x)$ is the risk of death at age $x$, which is independent of the birth year. The left term on the right side is the excess death risk that represents a person's risk of mortality from mesothelioma by asbestos exposure. The term consists of a coefficient and the annual product between birth and death. The product contains $c(a)$, the exposure concentration at a given age and power of period from age $a$ to the age at death. Thus, it is dependent both on age at death and exposure concentration to asbestos. The right term on the right side of equation 1 is the background risk occurred spontaneously, which depends only on age. The Banaei model differs from traditional models in Japan and other countries in that includes the initial age at exposure, the exposure period, and the annual amount of imported asbestos as a parameter. It is appropriate to predict the future trends in mesothelioma mortality by this model, but it is assumed that several alterations will be needed when applying this model to Japan. Hence, we developed a novel model for estimation of mesothelioma mortality in Japan based on the Banaei model. This model is also based on several hypotheses as follows: 
- Each group of this model consists of individuals born in the same year and mesothelioma fatality is independent of the age at death.

- Mesothelioma is caused just by occupational asbestos exposure.

- Once people are exposed to asbestos, they are at risk of death from mesothelioma.

- The risk increases with a greater exposure to asbestos.

- The risk function for mesothelioma is the sum of the annual risks from the beginning to the end of each year of asbestos exposure; the periodic risk is obtained by multiplying the term risk from asbestos exposure to death by the population rate of asbestos exposure each year.

- The population exposed to asbestos is proportional to the product of labor force by age and the relative amount of asbestos imported per year in manufacturing industry and construction industry.

- Every group was exposed to asbestos at the same age. Their exposure ceased at the same age as well.

- The values of several parameters are resolved by including the actual number of mesothelioma deaths.

On the basis of these criteria, we derived equation 2,

$R(y, x)=\sum_{a=A_{0}}^{a=A_{1}} s(y, a) f(x, a)$

where the symbols are defined as follows:

$y$ : the birth year

$x$ : the age at death from mesothelioma

$R(y, x)$ : the risk that cohort born in year $y$ die from mesothelioma at age $x$

a: age

$A_{0}$ : age at initial exposure

$A_{1}$ : age at end of exposure

$s(y, a)$ : the population rate of asbestos exposure at age $a$ of cohort born in year $y$

$f(x, a)$ : the term risk from exposure to death.

Equation 3 is the population rate of asbestos exposure, $s(y, a)$,

$s(y, a)=\frac{L(y, a) V(y+a)}{P(y, a)}$

where the symbols are defined as follows:

$L(y, a)$ : the size of the labor force in manufacturing industry and construction industry at age $a$ of cohort born in year $y$

$V(y+a)$ : the relative value of imported asbestos in year $(y+a)$ $P(y, a)$ : the population at age $a$ of cohort born in year $y$.

Equation 2 gives the total mesothelioma risk for a person exposed to asbestos, which is the yearly sum of age at initial to final asbestos exposure. The annual risk is derived by multiplying the population rate of asbestos exposure at a given age by the term risk from the initial to final age at exposure. From equation 3, the population rate of asbestos exposure is given by a value derived from multiplying the size of the labor force in manufacturing industry and construction industry by the relative amount of imported asbestos, and dividing by the population by age. The relative amount of imported asbestos is different each year, and reaches a maximum value of 1 .

Our model differs from the Banaei model in the following ways: We used people aged $50-74 \mathrm{yr}$ as the target of this model, whereas the Banaei model used men aged 50-79 yr. We limited our analysis to people who have had occupational exposure to asbestos, whereas the Banaei model included all men. As per the MOE survey, $80 \%$ of all mesothelioma deaths in Japan used asbestos at work ${ }^{12)}$. Thus, we considered it feasible to restrict the cause of mesothelioma to occupational asbestos exposure. We calculated the actual number of mesothelioma deaths to elucidate parameters causing $80 \%$ of all deaths. The Banaei model hypothesized only 2 mesothelioma causes: one caused by asbestos exposure and the other having a spontaneous origin. The latter means that even people unexposed to asbestos are at a risk, albeit a low one, of developing mesothelioma. Although an estimate of mesothelioma deaths before the advent of asbestos use is required to gauge the risk of a spontaneous onset, the Japanese government estimated the number after 1979 using the International Classification of Diseases, Ninth Revision (ICD-9). As Japan has been an asbestos importer since 1949, after World War II, it is difficult to estimate the unexposed risk. In addition, few studies exist that indicate a different risk between the Europeans and Japanese to spontaneously develop mesothelioma. Hence, we used the values that Banaei estimated, $K_{b}=1.5 \times 10^{-12}$ and $P_{b}$ $=3.5$. However, we disregarded these values because the spontaneous risk turned out to be very low in comparison with the total risk after the calculations.

We estimated the future trend for 2003-2050. Since 1995, annual mesothelioma deaths have been compiled in 5 age bands, as in the International Classification of Diseases, Tenth Revision (ICD-10). According to a nationwide questionnaire survey on clinical and pathological cases of malignant mesothelioma from 1995 through 2002, the immunohistological staining method had not sufficiently been used as a diagnostic technique of the mesothelioma and the researchers reported the results to all 
responders ${ }^{13,14)}$. Hence, we assumed the published mortality to be statistically authoritative after 2003 owing to the difficulties posed by the lack of technical expertise prior to this year. Taking into account the period of asbestos use and the time period from asbestos exposure to disease diagnosis, we assumed that the group that has been exposed to asbestos will not die of mesothelioma after 2050 .

\section{Risk function}

Based on the epidemiological research of Peto et al. ${ }^{10)}$, who investigated asbestos workers under $80 \mathrm{yr}$, the Banaei model shows that the term risk, $f(x, a)$, is exponentionally proportional to the years from exposure to death. Nicholson's study reported that the mesothelioma mortality increased gradually from exposure for a certain period and then decreased ${ }^{15)}$. We, thus, believe that $f(x, a)$, which is the risk during the period from asbestos exposure to death from mesothelioma may be applied into a lambda function, the monotonic increase and decrease on the boundary of a certain value. We substituted $f(x, a)$ for this expression which is multiplied exponentationly by attenuation as used by Berry ${ }^{9)}$.

$f(x, a)=K_{e} e^{-(x-a) \lambda}(x-a)^{P_{e}}$

where the symbols are defined as follows:

$K_{e}$ : the coefficient for mesothelioma mortality from asbestos exposure;

$P_{e}$ : the exponent for the age at mesothelioma mortality from asbestos exposure;

$\lambda$ : the constant for exponent

We can derive this equation from the Banaei Model by substituting $\lambda$ for " 0 ".

\section{Estimation of mesothelioma mortality}

The following describes the values assigned to this model:

- Relative value of imported asbestos $V(t)$

The Ministry of Finance has published the annual amount of asbestos imported since 1930 in the Trade Statistics of Japan. The Japanese Government started to import asbestos in the beginning of 1900s and stopped during World War $\mathrm{II}^{3}$. Hence, there is only a very low possibility that people dying of mesothelioma after 2003 were exposed to asbestos before or during the war. In this study, we used the data from 1949 when Japan resumed asbestos imports. $V(t)$ ranges between 0 and $1 . V(t)$ is given 1 as a maximum in 1974 when the highest amount of asbestos was imported post-World War II. And then, $V(t)$ at $t$ year is given as a relative value from asbestos import in 1974.
- Labor force of manufacturing industry and construction industry $L(y, a)$ [in thousands of people]

We used the labor force survey since 1949 released by the Ministry of Internal Affairs and Communications $(\mathrm{MIAC})^{16)}$. This survey divides the labor force into 5 age bands and by type of business in all ages per annum. As the labor force data stratified by age and profession is not available, we made the following revision: we calculated the proportion of working people employed in manufacturing industry and construction industry to all Japanese working population, then multiplied the rate and labor force by the 5 age bands together, and then divided the resulting value by 5 .

- Population by age $P(y, a)$ [in thousands people]

We used the population by age up to 2007 from the Census ${ }^{17)}$ and population estimate ${ }^{18)}$ published by the Statistics Bureau of MIAC. We used the Population Statistics of Japan ${ }^{19)}$ after 2008 with the median fertility and median mortality variants as published by the National Institute of Population and Social Society Research.

- Risk of period from the time of initial exposure to death $f(x, a)$

As in the preceding statement, we used $K_{e} e^{-(x-a) \lambda}(x-a)^{P_{e}}$. - Age at initial $A_{0}$ and final $A_{1}$ exposures

We applied these values using the data for $221 \mathrm{pa}$ tients $^{20)}$ who were diagnosed with mesothelioma by clinical and pathological examination in 27 hospitals from January 2000-2008. Table 1 indicates the period of asbestos exposure, the age at initial asbestos exposure and the latency period of these 221 persons. The values were processed statistically, because it was difficult to get individual data in view to protect personal information. We regarded getting mesothelioma to be the same as the date of death, because the disease has a very low 5-yr survival rate and a short survival time. Table 2 indicates average values calculated from Table 1 . Our model used $A_{0}=24$ and $\mathrm{A}_{1}=51$ as approximate values.

Optimal $K_{e}, P_{e}$, and $\lambda$ values were calculated by including actual mesothelioma deaths from 2003 to 2009. The MOE survey suggested that $80 \%$ of mesothelioma cases in Japan were attributable to occupational asbestos exposure. Hence, $80 \%$ of all mesothelioma mortality is applicable to this model. In addition, the optimum figure minimizes the residual error by the sum of squares between the actual mortality and estimated mortality population divided into 5 age bands. In this study, there are 40 possible combinations, because the future trend of mortality from 50 to 74 yr of age is estimated. 
Table 1. Period of asbestos exposure, the age at initial asbestos exposure and the latency period

\begin{tabular}{llcccccr}
\hline Region & Survey item & Number & Min & Max & Med & Ave & SD \\
\hline \multirow{2}{*}{ Pleura } & Period of asbestos exposure [yr] & 144 & 1 & 55 & 30 & 27.3 & 14.8 \\
& Age at initial asbestos exposure & 140 & 15 & 50 & 21 & 23.7 & 8.0 \\
& Latency period [yr] & 143 & 14 & 64 & 43 & 42.6 & 9.5 \\
& Peritoneum & 18 & 6 & 52 & 21 & 26.8 & 15.6 \\
& Period of asbestos exposure [yr] & 17 & 16 & 35 & 21 & 22.9 & 5.5 \\
& Age at initial asbestos exposure & 17 & 28 & 62 & 45 & 43.4 & 8.8 \\
\multirow{5}{*}{ Both } & Latency period [yr] & 164 & 1 & 55 & 30 & 27.6 & 14.9 \\
& Period of asbestos exposure [yr] & 159 & 15 & 50 & 21 & 23.6 & 7.8 \\
& Age at initial asbestos exposure & 162 & 14 & 64 & 43 & 42.5 & 9.5 \\
\hline
\end{tabular}

SD: standard deviation.

Table 3. Estimated and real death number after substituting each parameter (2003-2009)

\begin{tabular}{lccccccccccccc}
\hline & & Ke & Pe & 2003 & 2004 & 2005 & 2006 & 2007 & 2008 & $\begin{array}{c}2009 \\
\text { Sum }\end{array}$ & $\begin{array}{c}\text { Least-square } \\
\text { value }\end{array}$ \\
\hline Actual death number & & & - & - & 410 & 453 & 414 & 480 & 474 & 544 & 507 & 3,281 & - \\
Estimation death number & Model 1 & $3.43 \times 10^{-32}$ & 25.54 & 449 & 461 & 473 & 481 & 485 & 486 & 487 & 3,323 & 22,045 \\
& Model 2 & $6.00 \times 10^{-30}$ & 22.03 & 406 & 430 & 454 & 474 & 489 & 501 & 511 & 3,265 & 7,693 & 5,23 \\
& Model 3 & $1.58 \times 10^{-21}$ & 13.77 & 391 & 417 & 444 & 469 & 489 & 507 & 523 & 3,241 & 5,115 \\
& Model 4 & $1.20 \times 10^{-17}$ & 10.06 & 389 & 415 & 443 & 468 & 490 & 509 & 528 & 3,241 & 4,531 & 431 \\
& Model 5 & $9.30 \times 10^{-16}$ & 8.25 & 388 & 414 & 442 & 468 & 490 & 510 & 530 & 3,242 & 4,303 \\
& Model 6 & $1.18 \times 10^{-14}$ & 7.20 & 387 & 414 & 442 & 468 & 491 & 511 & 531 & 3,243 & 4,186 \\
& Model 7 & $6.36 \times 10^{-14}$ & 6.50 & 387 & 413 & 441 & 468 & 491 & 512 & 532 & 3,245 & 4,118 \\
& Model 8 & $1.90 \times 10^{-10}$ & 3.17 & 387 & 413 & 441 & 469 & 493 & 514 & 536 & 3,253 & 3,886 \\
\hline
\end{tabular}

Table 4. Total estimated number of mesothelioma death and peak year from 2003 to 2050 (50-74 ages / only occupational asbestos exposure)

\begin{tabular}{lcccccccc}
\hline & Model 1 & Model 2 & Model 3 & Model 4 & Model 5 & Model 6 & Model 7 & Model 8 \\
\hline Peak year & 2010 & 2015 & 2015 & 2016 & 2016 & 2016 & 2016 & 2016 \\
Death number & 11,827 & 14,081 & 15,530 & 16,127 & 16,441 & 16,634 & 16,761 & 17,443 \\
\hline
\end{tabular}

Table 2. Age at initial $A_{0}$ and final $A_{1}$ exposures

\begin{tabular}{lc}
\hline & Average age \\
\hline Age at initial $A_{0}$ exposure & 23.6 \\
Age at final $A_{1}$ exposure & 51.2 \\
\hline
\end{tabular}

\section{Results}

Optimal values for $K_{e}, P_{e}$, and $\lambda$ using 8 scenarios were calculated. In the first scenario, the peak year of $f(x, a)$ used is $30 \mathrm{yr}$ after initial exposure (model 1). The second scenario uses $35 \mathrm{yr}$ (model 2), the third, 40 (model 3), the fourth, 45 (model 4), the fifth, 50 (model 5), the sixth, 55 (model 6), the seventh, 60 (model 7); and the eighth scenario is that decay is not assigned $(\lambda=0)$ in equation 4 (model 8). Table 3 indicates $K_{e}, P_{e}$, the least-square values and the figures. The actual death number is multiplied 0.8 times by the number of people dying from mesothelioma. Significant differences were not found among any of the models. In addition, the total mesothelioma mortality between 50 and $74 \mathrm{yr}$ of age from 2003 to 2050 was calculated. Table 4 indicates the estimated number and peak year, which will come around 2015 using any of the models. The number of deaths ranges from 11,827 to 17,443 .

\section{Discussion}

\section{Mortality estimation}

The target subjects of this research were males and females aged 50-74 yr. A lower bound was set at $50 \mathrm{yr}$ due 
Table 5. Total estimated number of mesothelioma death and peak year from 2003 to 2050 (50-89 ages / only occupational asbestos exposure)

\begin{tabular}{lcccccccc}
\hline & Model 1 & Model 2 & Model 3 & Model 4 & Model 5 & Model 6 & Model 7 & Model 8 \\
\hline Peak year & 2015 & 2017 & 2020 & 2022 & 2023 & 2024 & 2024 & 2027 \\
Death number & 15,948 & 22,840 & 30,033 & 34,784 & 37,963 & 40,223 & 41,876 & 53,062 \\
\hline
\end{tabular}

Table 6. Concentration coefficient $r$ related to asbestos regulation

\begin{tabular}{lcl}
\hline \multicolumn{1}{c}{ Year } & $\begin{array}{c}\text { Coefficient } \\
\text { value }\end{array}$ & \multicolumn{1}{c}{ Regulated concentration } \\
\hline Before 1971 & 1 & No regulation \\
$1972-1974$ & 1 & $2 \mathrm{mg} / \mathrm{cm}^{3}\left(33\right.$ fibers $\left./ \mathrm{cm}^{3}\right)$ since 1972 as asbestos inhibitory concentration \\
$1975-1987$ & $5 / 33$ & $5 \mathrm{fibers} / \mathrm{cm}^{3}$ since 1975 as asbestos inhibitory concentration \\
$1988-2003$ & $2 / 33$ & 2 fibers $/ \mathrm{cm}^{3}$ since 1988 as administrative level \\
After 2004 & $0.15 / 33$ & $0.15 \mathrm{fibers} / \mathrm{cm}^{3}$ since 2004 as administrative level \\
\hline
\end{tabular}

to the difficulty and unreliability in mesothelioma diagnoses for the earlier and middle decades of life. According to the statistics from Ministry of Health, Labour and Welfare, the number of mesothelioma deaths in ages $50-74$ and $50-89$ yr account for 54.8 and $94.5 \%$ of total deaths ${ }^{2)}$. In addition, as the aged population in Japan shows a higher prevalence of mesothelioma, we should have estimated by eliminating the data from people aged over $75 \mathrm{yr}$. This model estimates the ages at initial and final exposures, and annual risk is additive from $A_{0}-A_{1}$. Those were born before 1925 and attained $24 \mathrm{yr}$ of age in 1949 did not have an exposure risk before 1948. That cohort may not be quantified, and would reduce the result because the added frequency is less than that of the other cohorts. For group aged over $75 \mathrm{yr}$, which adds up to 0 in some years, reliable values could not be worked out.

Furthermore, it is crucial to include people aged over $75 \mathrm{yr}$ when we project mesothelioma deaths until 2050. Expanding the target of patients as a 50-89 yr age bracket, we attempted to calculate the projections for mesothelioma deaths by using this model. However, a clear difference is observed between the estimated and real number in any generation, especially for the 80 -yr-olds. Although a large difference between the estimated and actual result for people aged over $80 \mathrm{yr}$ is observed, we assessed the total population dying from mesothelioma aged $50-89$ yr by using this model. The results are shown in Table 5.

This model is based on occupational asbestos exposure. The MOE survey reported that approximately $80 \%$ of mesothelioma deaths were attributable to occupational asbestos exposure ${ }^{12)}$. The total correct mortality is found by multiplying the calculated number by 1.25 ; and we estimated the range as 19,935-66,327. The maximum pro- jected death toll, which has a wide range, is over 66,000; that number is much lower than Murayama's estimate ${ }^{5)}$. The difference cannot be accounted for as the study population and the data used are different in both studies. While an age-cohort model is used to estimate future trends from past death numbers and population only, our model used not only those numbers but also the quantities of asbestos imported and the size of the labor force.

\section{Potential impact of regulatory compliance}

European countries, which began utilizing large amounts of asbestos prior to its advent in Japan and incurred asbestos-related diseases initially, also took measures to regulate asbestos use prior to Japan. The Japanese government has regulated asbestos use since 1972 following the guidelines in European countries. However, it seems that adequate ventilation and equipment to protect from asbestos exposure had not been used in the workplaces where asbestos use was rampant. Hence, we attempted to estimate the figure of adequately regulated workplaces.

This research targeted occupational exposure to asbestos and thus we focused on the administrative level or asbestos inhibitory concentration that is predetermined by the Industrial Safety and Health Act. We added equation 4 to concentration coefficient $r$, as shown in Table 6 , concerned with regulation as equation 5 .

$R(y, x)=K_{e} \sum_{a=A_{0}}^{a=A_{1}} \frac{L(y, a) V(y+a)}{P(y, a)} r(y+a) e^{-(x-a) \lambda}(x-a)^{P_{e}}$

We set the coefficient value of 1 according to the Industrial Safety and Health Act implemented in 1972 and 


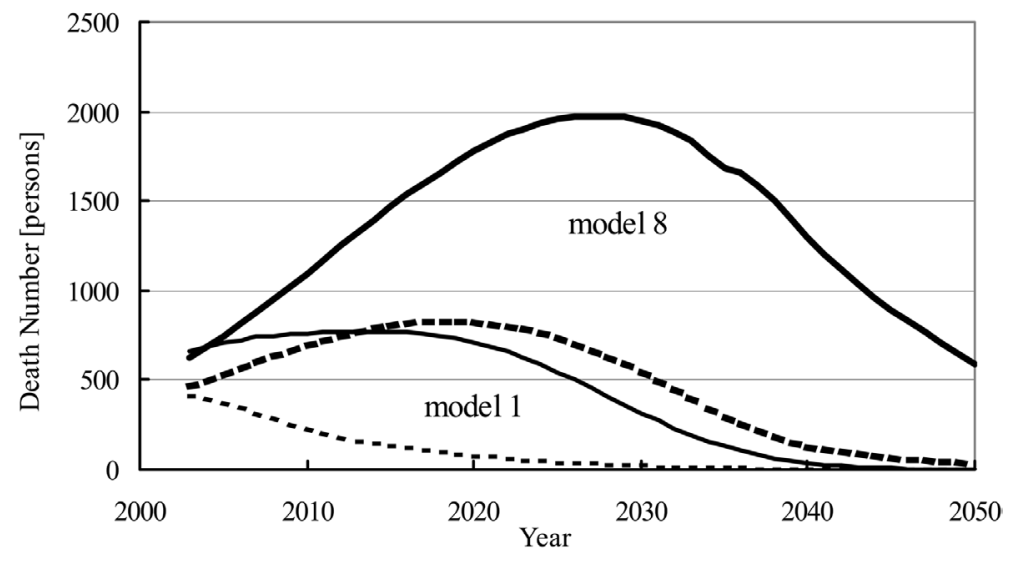

Fig. 2. Estimated death number of mesothelioma in case of regulatory compliance and noncompliance (2003-2050 yr, 50-89 age, model 1 and 8).

took the relative value from the revised asbestos inhibitory concentration after the regulation.

Assigning the values of Table 6 to equation 5, another mortality figure is calculated that includes an offset of non-occupational exposure. We had 2 scenarios, model 8 and model 1 . The former provided maximum and the latter minimum estimates for mortality. Consequently, the peak year was found to be 2019, and the estimated number of total deaths as 23,463 by 2050 in model 8 of equation 5 . For model 1, the peak had already been reached in 2003; the annual death number showed a linear decrease and a total estimate of 4,394. Figure 2 indicates the results: the heavy line indicates calculations from model 8 , and the narrow line, from model 1 . The case for regulatory compliance and that for noncompliance are represented by is the dotted and solid lines, respectively. This indicates that more than $60 \%$ of deaths will be avoided if the regulations are followed as compared with the case when they are breached. There is no doubt that preserving the administrative level in the workplace in which asbestos used would be sufficiently effective to reduce the present and future deaths from mesothelioma.

\section{Limitations and uncertainties}

In this study, data limited to $8 \mathrm{yr}$ are expanded to the estimated expected deaths over 5 decades due to a lack of technical expertise at the time. More reliable estimates can be generated in future when further data for mesothelioma mortality become available. In addition, chrysotile, amosite, and crocidolite were mainly used in Japan, and these imports have not been recorded. The relative risk for mesothelioma mortality from crocidolite and amosite asbestos is greater than that from chrysotile asbestos is by some 500 and 100 times, respectively ${ }^{21)}$. Imports of amosite and crocidolite were stopped through industrial voluntary regulation in 1989; it is probable that chrysotile, which has relatively low carcinogenicity, would have been used after that year. Considering that, future mortality may actually be lower than our projections.

In the meantime, this investigation targeted only those who had occupational exposure to asbestos; we do not consider exposure through demolition of buildings and disposal of building materials in which asbestos was used. Exposure to asbestos is expected to occur not only through usage in the workplace, but also during teardown or disposal of building materials. After a strong earthquake occurred in Kobe in 1995, both processors of building materials and the general population were exposed to asbestos. Our estimate does not consider exposures to asbestos from those sources.

\section{Conclusion}

Limited available estimates reveal future trends for mesothelioma mortality resulting from asbestos exposure in Japan. The number of mesothelioma deaths from occupational asbestos exposure is estimated to be $15,948-53,062$ in 2003-2050. In addition, the number of mesothelioma deaths resulting from all kinds of exposure has is estimated to be 19,935-66,327 in the years 2003-2050. Our estimate suggests that the number of mesothelioma deaths could be significantly reduced if there were adequate compliance with the administrative level guidelines for occupational asbestos exposure. Future studies that stress on education with regard to protection from exposure during the demolition or the disposal of asbestos-containing materials, and 
more adequate measures for reducing mesothelioma risk are needed.

\section{References}

1) Gardner MJ, Saracci R (1989) Effects on health of nonoccupational exposure to airborne mineral fibers. IARC Sci Publ 90, 375-97. [Medline]

2) Japan Ministry of Health, Labour and Welfare (2008) Annual trend on mesothelioma mortality to prefectures in 1995-2007: from Vital Statistics. http://www.mhlw.go.jp/ toukei/saikin/hw/jinkou/tokusyu/chuuhisyu07/index.html. Accessed December 28, 2009 (in Japanese).

3) Japan Asbestos Association (2007) Japanese asbestos imports. Japan Asbestos Association, Tokyo (in Japanese).

4) House of Representatives (2008) Survey on execution status of laws related to asbestos in 2007, Research Office on Environment Research Bureau House of Representatives, Tokyo (in Japanese).

5) Murayama T, Takahashi K, Natori Y, Kurumatani N (2006) Estimation of future mortality from pleural malignant mesothelioma in Japan based on an age-cohort model. Am J Ind Med 49, 1-7. [Medline] [CrossRef]

6) Tossavainen A (2004) Global use of asbestos and the incidence of mesothelioma. Int J Occup Environ Health 10, 22-5. [Medline]

7) Banaei A, Auverta B, Goldberga M, Gueguena A, Lucea D, Goldberga S (2000) Future trends in mortality of French men from mesothelioma. Occup Environ Med 57, 488-94. [Medline] [CrossRef]

8) Peto J (1979) Dose-response relationships for asbestosrelated disease: implications for hygiene standards. Part II. Mortality. Ann N Y Acad Sci 330, 195-203. [Medline] [CrossRef]

9) Berry G (1991) Prediction of mesothelioma, lung cancer, and asbestosis in former Wittenoom asbestos workers. Br J Ind Med 48, 793-802. [Medline]

10) Peto J, Seidman H, Selikoff IJ (1982) Mesothelioma mortality in asbestos workers: implications for models of carcinogenesis and risk assessment. Br J Cancer 45, 124-35. [Medline] [CrossRef]
11) Hughes JM, Weill H (1986) Asbestos exposure: quantitative assessment of risk. Am Rev Respir Dis 133, 5-13. [Medline]

12) Azuma K, Uchiyama I, Chiba Y, Okumura J (2009) Mesothelioma risk and environmental exposure to asbestos. Int J Occup Environ Health 15, 166-72. [Medline]

13) Morinaga K, Iuchi Y, Bessho A, Nakano K, Okumra S, Higashiyama M, Nakano K, Hiraki A (2004) Study on improvement in diagnostic accuracy and treatment of malignant mesothelioma. Annual Report of the Cancer Research, Ministry of Health, Labour and Welfare, 448-52, National Cancer Center, Tokyo (in Japanese).

14) Morinaga $K$, Iuchi $Y$, Bessho $A$, Okumura $S$, Higashiyama M, Nakano K, Aoe K, Kamei T (2005) Study on improvement in diagnostic accuracy and treatment of malignant pleural mesothelioma. Annual Report of the Cancer Research, Ministry of Health, Labour and Welfare, 340-4, National Cancer Center, Tokyo (in Japanese).

15) Nicholson WJ (1976) Recent approaches to the control of carcinogenic exposures. Case study1: asbestos - the TLV approach. Ann N Y Acad Sci 271, 152-69. [Medline] [CrossRef]

16) Ministry of Internal Affairs and Communications. Labour Force Survey. Statistics Bureau, Ministry of Internal Affairs and Communications, Tokyo (in Japanese).

17) Ministry of Internal Affairs and Communications. Population Census. Statistics Bureau, Ministry of Internal Affairs and Communications, Tokyo (in Japanese).

18) Ministry of Internal Affairs and Communications. Population Estimates. Statistics Bureau, Ministry of Internal Affairs and Communications, Tokyo (in Japanese).

19) National Institute of Population and Social Security Research (2008) Population Statistics of Japan 2008. National Institute of Population and Social Security Research, Tokyo (in Japanese).

20) Usami I (2009) Surveillance study on mesothelioma due to asbestos exposure in Japan, clinical study of 221 cases in hospitals for workmen's compensation. Research Center for Asbestos-related Diseases, Japan Labour Health and Welfare Organization, Tokyo (in Japanese).

21) Hodgson JT, Darnton A (2000) The quantitative risks of mesothelioma and lung cancer in relation to asbestos exposure. Ann Occup Hyg 44, 565-601. [Medline] 